\title{
A control system that causes the check for obstruction of the ducts of seed metering
}

\author{
Lukasz Gierz ${ }^{1, *}$, Żaneta Staszak $^{1}$, Dawid Wojcieszak ${ }^{2}$, Jan Szymenderski ${ }^{3}$, Jacek \\ Marcinkiewicz ${ }^{1}$, Eukasz Semkło and Bartłomiej Paszkiewicz ${ }^{5}$ \\ ${ }^{1}$ Poznan University of Technology, Institute of Machines and Motor Vehicles, Poznań, Poland \\ ${ }^{2}$ Poznan University of Life Sciences, Faculty of Agronomy and Bioengineering, Poznań, Poland \\ ${ }^{3}$ Poznan University of Technology, Institute of Electrical Engineering and Electronics, Poznań, \\ Poland \\ ${ }^{4}$ Chair of Thermal Engineering, Poznan University of Technology, Poznań, Poland \\ ${ }^{5}$ Wroclaw University of Science and Technology, Faculty of Microsystem Electronics and Photonics, \\ Wrocław, Poland
}

\begin{abstract}
Even sowing of the seeds is very important because the quality of the crop depends on it. For this reason, the aim is to sow without a culvert caused by an obstruction of the seed hose. For this purpose, a patency control system has been developed for hoses that allow the seed to enter the ground. This allows to ensure the uniformity of seeding, which is very desirable nowadays. The developed method consists in mounting parallel sensors built into the head or on the output nozzles from the head and sensors in the coulters from which information will be transmitted to the monitoring system.
\end{abstract}

\section{Introduction}

The development of agricultural mechanization focuses on increasingly efficient use of available land for crop production. Each year, the area under cultivation is reduced as it is converted into investment and construction for the development of the world's population. This development of the economy makes the cities grow, and thus the demand for food increases. Hence the need for innovation in agriculture - it is necessary to use the most of every available area. One of the factors influencing the increase in yield is the accuracy of sowing. For greater efficiency, research is being carried out into the development of machine design to ensure that seeding is close to the ideal. This means that ideal distances should be maintained and the seeds should be evenly distributed over the surface. For this purpose, it was decided to install special piezoelectric sensors in the pneumatic hoses of the pneumatic seeder to monitor the flow of seeds and verify their passability. In order to calibrate the machine consisting of a sensor and a system for analysing signals and displaying results, it is necessary to know the speed of the grains moving in the seeding

\footnotetext{
* Corresponding author: lukasz.gierz@put.poznan.pl
} 
hoses. An interesting solution for measuring the speed of grain was proposed in the Industrial Institute of Agricultural Machinery in Poznań [1]. The author presented the method of determining the peripheral velocity of grain and its qualification by means of a vision system. In order to correctly determine all parameters affecting grain flow, it is necessary to know its size and shape, which contributes to the use of vision systems which, based on image analysis, identify the cereal variety and its shape $[2,3]$. Other solutions are piezoelectric, microphone or photoelectric sensors $[4,5,6]$. Previously, the piezoelectric touch transducer was used in the keyboard of electronic devices, where the low pressure was converted into electrical voltage [7]. The piezoelectric sensor was also used to measure the engine speed [8]. In addition, piezoelectric transducers are also used to evaluate the restitution factor, the degree of seed damage and the amount of energy damaging the grain $[9,10]$. The solutions of yield loss control or yield monitoring systems are also known [11, 12]. In this system, two different sensors were used to count the yield obtained during the harvest of cereals by a combine harvester. The yield is determined on the basis of a combination of signals from the first and the second sensor.

\section{Material and Research Methods}

Within the Lider project, an attempt was made to develop a control system of seeding obstruction that can be used in mechanical-pneumatic seeders and as an indicator of crop losses. As mentioned earlier, agricultural development contributes to the use and construction of more productive machinery. In the case of seed drills, there is a problem with the control of the sowing rate quantity and interruptions in the sowing rate due to the obstruction of the seed hoses or coulters. Optical sensors of yield monitoring are commonly used in pneumatic seeders, but they do not have the possibility to control the passability in dusty places near the ground, e.g. in coulters. The proposed system uses a PVDF sensor in the form of a strip of foil, enabling the development of low-cost sensors available to all manufacturers of agricultural machinery. An innovative solution is to use simultaneously control sensors (counting the grains) inside the distributor head and in the sowing coulter. This solution allows to achieve the following benefits:

- possibility of mutual verification of counted grains of both sensors,

-improved reliability through system paralleling,

-if the control system is combined with the seed drill control system, the seed rate can be set automatically.

The use of two sensors will contribute to a smaller measurement error of the quantity of seeds and a more accurate self-adjustment. The developed seeding obstruction control system includes sensors (Fig. 1) with piezoelectric foil placed in the coulters and sensors of distribution heads connected in a wired or wireless way to the central control unit. Head sensors are built into the heads or placed on the outlet nozzles from the heads, while in the case of universal seed drills the sensors are placed on the outlet from the seed box.

The head sensors are made of shielded piezoelectric foil (2) placed on a damping material (1) mounted on a base made of polyurethane foam with a thickness of 2.5 to 3.5 $\mathrm{mm}$. The whole is then covered with a rubber casing (4) with a thickness of 1.5 to $2 \mathrm{~mm}$ to prevent damage. The distribution cone of the distributor head (3) consists of segments arranged in such a way that the angle of release of the seed is less than $45^{\circ}$ and the tip of the cone is rounded to minimise seed damage. In addition, if the display contains a wi-fi or Bluetooth communication interface, a connection to the control unit is possible. 


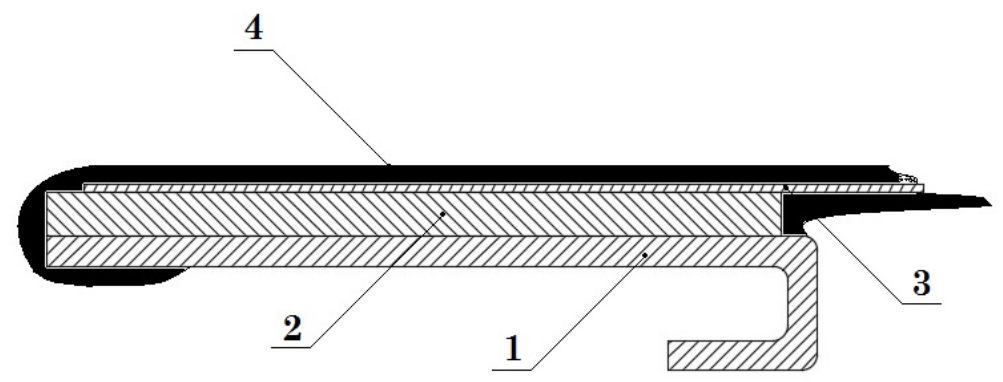

Fig. 1. Monitoring and obstruction sensor - cross-section of the structure [own elaboration]

In the developed seeding obstruction control system, sensors were placed in each coulter and 16 sensors in the head, optionally in the output nozzles from the head or on the output from the seed box (Fig. 2).

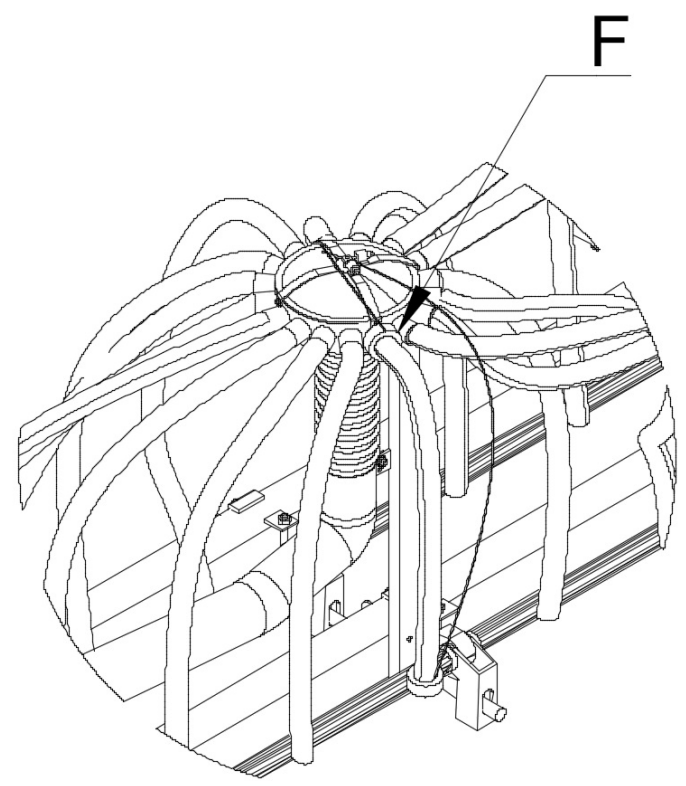

Fig 2. Distribution head F with monitoring and obstruction sensors mounted on the output nozzles from the head [own elaboration]

When placing the sensors in the output nozzles, there is no need to interfere with the construction of the distributor head, because they can be made as connectors (extensions) of the distributor head with a pneumatic hose by various methods, e.g. by rapid prototyping ( $3 \mathrm{D}$ printing) or injection method. At such a location of the sensor, the grain hits the sensor only after it bounces off the opposite wall of the seed hose, which reduces the signal strength. This design of the seeding obstruction control system can be used especially in mechanical, mechanical-pneumatic, pneumatic and combine harvesters, but also for other technological systems, in which it is necessary to monitor the flow of grain material. In addition, the seeding obstruction control system makes it possible to retrofit existing control systems for seed drills and other equipment. The principle of the seeding obstruction control system is to obtain suitably filtered signals 
indicating the flow of a given number of grains or the lack of flow due to lack of signal. The control system (Fig. 3) consists of the parallel sensors of the coulter (marked in Fig. 3 as $\mathrm{C}$ and F) and the sensor 3. All elements of the system are placed in the coulters $\mathrm{B}$ and the distribution heads $G$ of the seed drill (alternatively in the seed hoses or at the outlet from the seed box) and are connected with the wires D or wirelessly with the central control unit A compatible with the display by means of the communication port $\mathrm{E}$ enabling the transmission of information by a wire or wireless means, placed in the $\mathrm{cab}$ of the tractor operator or on the seed drill itself from the control unit A. It also includes head sensors F connected to the central control unit A via wire D or wirelessly.

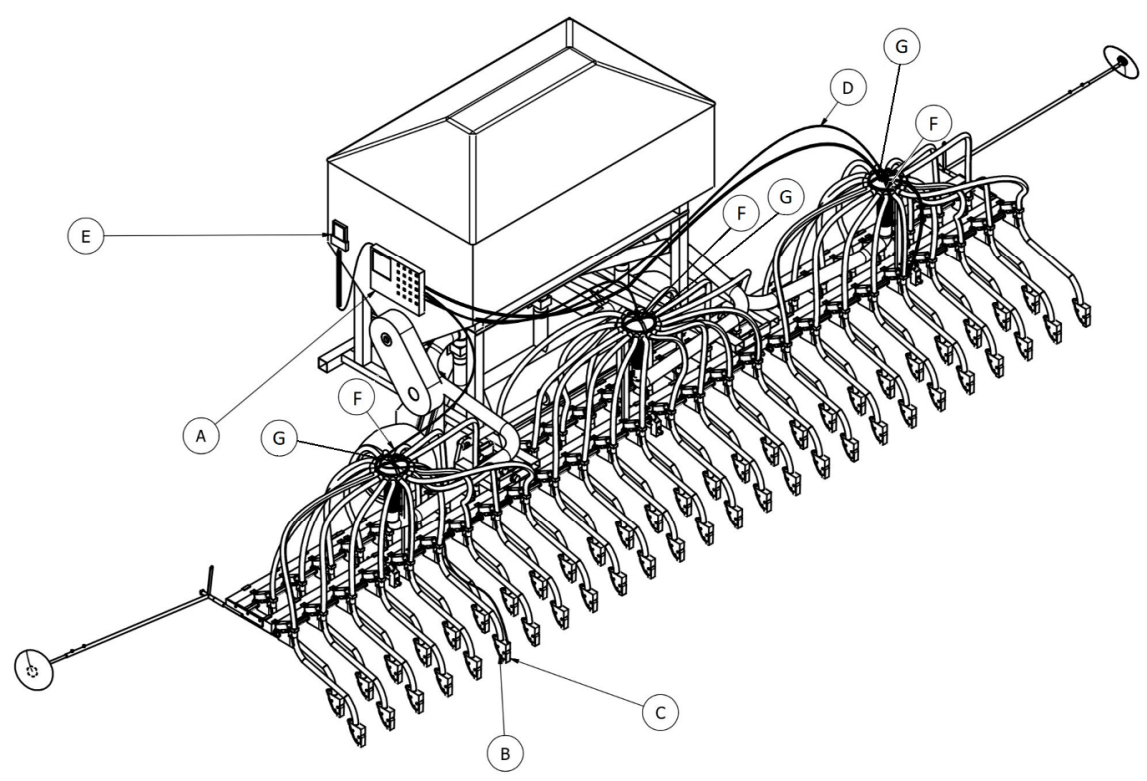

Fig 3. Obstruction control system with parallel sensors, i.e. sensors built into the head or on the output nozzles of the head, and the sensors in the coulters [own elaboration]

\section{Conclusions}

The developed system will contribute to increasing the uniformity of seed density by eliminating the unseeded area of the so-called culverts, and thus more accurate sowing will be carried out. This will ensure an increase in yield and a reduction in seed consumption, which is becoming more and more expensive. The seeding obstruction control system can be used in universal and mechanical-pneumatic seed drills for the sowing of cereals and other cultivated plants. It can also be used in systems where it is necessary to monitor the quantity or clogging of grain material as well as monitoring of crop losses, most often installed in combine harvesters.

\section{Acknowledgements}

The research was supported by the National Center for Research and Development under the LIDER VIII project number LIDER / 24/0137 / L-8/16 / NCBIR / 2017 


\section{References}

1. Z. Zbytek, Journal of Research and Applications in Agricultural Engineering, Poznań (2015).

2. Ł. Gierz, Journal of Research and Applications in Agricultural Engineering, Poznań (2011).

3. D. Karayel, M. Wiesehoff, A. Ozmerzi, J. Muller, Computers and Electronics in Agriculture, 50, (2006).

4. K. Ram Chandar, V. R. Sastry, C. Hegde, S. Shreedharan, Geomechanics and Geoengineering, 12 3, (2017)

5. H. Neopane, B. Thapa, Engineering and Technology Vol. 8, No. I (2012).

6. Y. Cheng-Ku, H. Pei-Rong, Atmos. Meas. Tech., 9 (2016).

7. F. J. Mazella, Measuring Motion with Imaging Software, NASA Tech Briefs, (2014)

8. M. Lambert, C. Froustey, J. L. Charles, J. L. Lataillade, Journal de Physique IV Vol. 110, Issue 1 (2003).

9. K. R. Rasmussen, M. Sørensen, JGR 113, (2008.)

10. I. Stancic, D. Borojevic, Human Kinematics Measuring Using a High Speed Camera and Active Markers, Laboratory for Biomechanics and Automatic Control Faculty of Electrical Engineering, Mechanical Engineering and Naval Architecture, University of Split (2001).

11. F. Cernuschi, C. Rothleitner, Powder Technology, 318, (2017).

12. K. Deguchi, Measurement, 4, Issue 4 (1986). 